\title{
Visual relational ("categorical") learning and performance by rats with temporal or partial striate cortical lesions
}

\author{
LAURA B. WILLIAMS and WILLIAM F. MCDANIEL \\ Georgia College and State University, Milledgeville, Georgia
}

\begin{abstract}
Previous research has shown that injuries of the inferotemporal cortex in monkeys result in impaired visual "categorical" perception. The goal of this project was to explore the possibility that a similar deficit might accompany injuries of the visual portions of the temporal cortex in rat, namely area TE2. Rats were prepared with bilateral TE2, partial striate, or sham cortical lesions and trained to discriminate between 17- (S+) and 3-cm (S-) diameter circles in a Y-shaped water maze. After criterion on this discrimination was reached, tests of relational perception were conducted with intermediate-size stimuli. The larger of the two stimulus pairs consistently cued the location of a submerged escape platform. Not only did rats with TE2 lesions learn the original discrimination as rapidly as normal rats and rats with partial striate lesions, but their performance paralleled that of the other groups on all tests of relational perception with intermediate stimuli. Several possible explanations for the discrepancy between the results observed here with rats and those observed with nonhuman primates are discussed.
\end{abstract}

It is now well established that two distinct cortical visual pathways exist in human (Haxby et al., 1991; Horwitz et al., 1992) and nonhuman (Macko et al., 1982; Mishkin, Ungerleider, \& Macko, 1983; Ungerleider \& Mishkin, 1982) primates. The dorsal occipital-parietalprefrontal pathway is specialized for visuospatial perception, whereas the ventral occipital-temporal-prefrontal pathway is specialized for complex pattern and object identification. Lesions within these regions produce severe dissociable deficits in visual-spatial and visual-memory tasks, respectively. Regional cerebral blood flow measurement revealed by positron emission tomography while humans performed a face-matching task or dot-location task has supported studies based on lesion by showing that the occipitotemporal cortex is involved in the visualmemory task whereas the posterior parietal cortex (PPC) is involved in visual-spatial localization (Haxby et al., 1991).

In a review of the scant literature on this topic in rat, Kolb (1990) hypothesized that a similar organization appeared to characterize the corticocortical projections emanating from the striate cortex of the rat. That is, an anterodorsal pathway arising from the striate and peristriate regions terminates in the PPC (Kolb \& Walkey, 1987), whereas a more ventrorostral pathway terminates in the visual-temporal region labeled TE2 (specifically, the

This research was supported in part by a Georgia College and State University faculty research grant to the second author. We thank David M. Compton for his assistance with several methodological and technical aspects of the project. Correspondence should be addressed to W. F. McDaniel, Department of Psychology, Georgia College and State University, Milledgeville, GA 31061-0490 (e-mail: bmcdanie@mail. gcsu.edu). more caudal regions of TE2; Shi \& Cassell, 1997). Neurobehavioral investigations have revealed that the PPC in rat plays an important role in spatial navigation on the basis of visual cues (e.g., Kolb, Buhrmann, McDonald, \& Sutherland, 1994; Kolb \& Walkey, 1987; McDaniel \& Thomas, 1978; McDaniel \& Wall, 1988), and that the PPC plays different but complementary roles in the two hemispheres in processing such cues (McDaniel et al., 1995; McDaniel, Williams, Attaway, \& Compton, 1998). Fewer studies have focused on functions of TE2 in rat. Davis and McDaniel (1993) prepared rats with bilateral PPC, TE2, or sham cortical ablations and trained the animals on a visual matching-to-sample problem and on a nonconceptual visuospatial conditional problem with the order of exposure to the tasks counterbalanced within groups. Animals with either type of lesion were inferior to the sham control animals on both tasks, and a dissociation between the lesion groups failed to emerge. Thus, although animals with TE2 lesions performed poorly on the visual-memory task, so did the animals with PPC lesions. Nevertheless, Kolb et al. (1994) conducted a similar study with what appears to be a simpler methodology and found, as is the case with primates, a dissociation between the influences of PPC and TE2 lesions on visualspatial and visual-memory functions. Animals with TE2 lesions were deficient in their visual matching-to-sample task, but not a spatial navigation task. The opposite was found with PPC-lesioned rats.

Recent investigations using electrophysiological methods and assays for Fos (i.e., the protein product of the immediate early gene $c$-fos) have supported the conclusion that area TE2 plays an important role in visual memory (Zhu, Brown, \& Aggleton, 1995; Zhu, McCabe, Aggleton, \& Brown, 1996). These investigations have shown 
that neurons in rat TE2 cortex are less responsive to familiar visual stimuli than they are to novel stimuli. Similar results had previously been described in primates (e.g., Sobotka \& Ringo, 1993). Also, they have shown that there is less intraneuronal c-fos activity in area TE2 when the animal is exposed to familiar visual objects than when exposed to novel objects. Similar results were observed in the perirhinal cortex, but not in any of the other areas studied (e.g., superior colliculus, occipital association cortex, entorhinal cortex, hippocampus, anterior cingulate gyrus, amygdala, etc.).

It has been reported that the temporal association cortex of humans and the inferotemporal (IT) cortex of monkeys plays an important role in "categorical" or relational visual perception (see Kolb \& Whishaw, 1990, p. 458). Wilson and DeBauche (1981) trained cynomolgous monkeys to discriminate between stimuli at two ends of a continuum in three separate problems. The problems consisted of a length discrimination task ( $10 \mathrm{vs} .55 \mathrm{~mm})$, a stripe orientation discrimination task $\left(0^{\circ}\right.$ vs. $\left.90^{\circ}\right)$, and a texture discrimination task ( 3.0 vs. 23.0 lines per $\mathrm{cm}$ ). The animals then received either IT or partial lateral striate (PS) cortical ablations, and they were tested with intermediate stimuli for both between- (e.g., with respect to the line length discrimination, $20 \mathrm{vs} .45 \mathrm{~mm}$ ) and within(e.g., $10 \mathrm{vs.} 25 \mathrm{~mm}$ ) category perception. Monkeys with IT lesions failed to demonstrate categorical perception, whereas normal control monkeys and monkeys with PS lesions demonstrated relatively good transfer of the preoperative habits to the postoperative discriminations with new stimuli. The authors concluded that "lacking IT cortex, monkeys tend to perceive visual stimuli more in terms of their physical values alone, and less in terms of their relationship to stimuli experienced previously in a perceptual domain" (p. 36).

The purpose of the present study was to explore the possibility that area TE2 in rat plays a necessary role in "categorical" or relational visual perception, as is the case in primates. Rats were prepared with bilateral TE2, PS, or sham cortical lesions and trained to discriminate a 17 $\mathrm{cm}$-diameter black circle $(\mathrm{S}+$ ) from a 3 -cm black circle $(\mathrm{S}-$ ). In several subsequent phases, generalization of the previously learned response to novel stimuli with intermediate diameters (e.g., $13 \mathrm{vs.} 7 \mathrm{~cm}$ ) was assessed. If the rat temporal cortex is important in "categorical perception," as appears to be the case for primates, then rats prepared with TE-2 lesions should perform as well as normal rats while learning the initial visual discrimination. However, TE-2 injured rats should exhibit considerable behavioral dysfunction on tests of visual relational perception. Three months after categorical perception was examined, long-term retention was explored by retraining the rats on the original discrimination.

Rats with PS lesions served as lesion control group to parallel the design in the study by Wilson and DeBauche (1981), where monkeys with PS lesions served as controls. Rats with PS lesions generally perform relatively well in tests of pattern discrimination, and it has been shown that even when prepared with total striate ablations, the destriate rat eventually masters demanding visual discriminations (e.g., Hughes, 1977; McDaniel \& Noble, 1984).

\section{METHOD}

\section{Subjects and Surgery}

Fifteen male Long-Evans rats, bred in our colony and ranging in age from 7 to 11 months at the beginning of the experiment, were used as subjects. Five rats were randomly assigned to each of three surgical conditions: PS, TE2, or sham control. Rats were individually housed in stainless steel rodent cages and entrained to a reversed light-dark cycle with light offset at 8:00 a.m. and onset at 8:00 p.m. All surgical and behavioral procedures were conducted during the dark phase. The animals were maintained on an ad-lib schedule of food and water. All aspects of the methodology were reviewed and approved by the University's Institutional Animal Care and Use Committee.

Each animal was weighed and administered an intraperitoneal injection of atropine $(.35 \mathrm{mg} / \mathrm{kg})$ and sodium pentobarbital $(45 \mathrm{mg} / \mathrm{kg})$. After the scalp was shaved and scrubbed with a $10 \%$ povidone-iodine solution, the animal was placed in a Kopf stereotaxic instrument. Using clean surgical instruments, a midline incision was made, the temporalis muscle was retracted ventrolaterally with a periosteal elevator, and the dorsal cranium was exposed.

For animals assigned to the sham control group, surgery was terminated at this point. The wound was closed with wound clips, and Mycitracin triple antibiotic ointment with lidocaine was applied liberally to the incision site.

For animals receiving PS lesions, the skull was trephined at an area $3.0 \mathrm{~mm}$ anterior to the Lambdoid suture and $3.0 \mathrm{~mm}$ lateral to the sagittal suture. Rongeurs were used to expand the area to expose area 17 (Hughes, 1977), and the underlying cortical tissue was aspirated. For animals receiving TE2 lesions, the skull was trephined $5.0 \mathrm{~mm}$ posterior to the coronal suture and 5.5 to $6.5 \mathrm{~mm}$ lateral to the sagittal suture. The craniotomy was enlarged several millimeters along the AP plane with a microrongeur to expose area TE2. Under visual guidance from a jeweler's loop $(3 \times$ magnification $)$ the dura was excised with a microdissection scalpel, and the target cortical mantle was aspirated through a glass micropipette. Following surgical procedures, the cavities were packed with Gelfoam and the wounds were closed as described for sham control animals. A 7-day postoperative recovery period was observed prior to initiating an evaluation of kinetic function. Training on the visual discrimination task began 13 days after surgery.

\section{Histology}

Upon completion of the behavioral procedures, PS and TE rats received an overdose of sodium pentobarbital, and they were perfused through the heart with $50 \mathrm{cc}$ of isotonic saline followed by $50 \mathrm{cc} 10 \%$ formalin. The brains were removed and stored in $10 \%$ formalin for 7 days prior to being photographed from the dorsal or lateral perspectives. Then they were transferred to a $30 \%$ sucrose solution in $10 \%$ formalin mixture and refrigerated for several days After being blocked to include the lesion site and thalamus, brains were frozen in a Cryocut II microtome and sectioned into $60-\mu \mathrm{m}$ slices through the coronal plane. Every fifth section was mounted on a slide and stained with cresyl-violet acetate. Sections were examined under a light microscope for evidence of retrograde degeneration in the thalamus, for accuracy of lesion placement, and for lesion depth.

\section{Apparatus and Stimuli}

The apparatus for examining visually guided behavior was a white, circular, galvanized steel water tank that measured $1.4 \mathrm{~m}$ in 
diameter and $0.6 \mathrm{~m}$ in height. The water tank was fitted with a Yshaped alley also constructed from galvanized steel. The $Y$ maze consisted of a stem, $86 \mathrm{~cm}$ long and $30 \mathrm{~cm}$ wide, with two arms, each $61 \mathrm{~cm}$ long and $54 \mathrm{~cm}$ wide. The apparatus was filled with water to a depth of $20 \mathrm{~cm}$. Water temperature was maintained at $26^{\circ}$ $\pm 1^{\circ} \mathrm{C}$. Bromide tablets were added approximately every 10 days to the water to discourage microbiological growth. White nontoxic paint (Artistica II tempera, Hazleton, PA) was added to the water to obscure view of a white escape platform $(26 \times 17 \times 17 \mathrm{~cm})$ submerged $2 \mathrm{~cm}$ below the water surface. Throughout all behavioral procedures, the platform was consistently located beneath the larger of two visual stimuli, and it was positioned adjacent to the rear wall of either $\mathrm{Y}$ arm of the apparatus. The distance from the start position to the escape platform was $140 \mathrm{~cm}$.

The visual stimuli consisted of eight, two-dimensional circles differing by increments of $2 \mathrm{~cm}(3,5,7,9,11,13,15$, or $17 \mathrm{~cm}$ in diameter). Each circle was solid black and centered on white paper $(33 \times 25 \mathrm{~cm})$. The circles were printed with nonwater soluble ink, and the stimuli were laminated. The stimuli were suspended from the rear wall of each of the two arms of the Y, and the bottom edges of the stimuli were approximately $2 \mathrm{~cm}$ above the water surface.

\section{Rotating Rod Test}

A wooden rod $(3.5 \mathrm{~cm}$ in diameter and $180 \mathrm{~cm}$ long) wrapped with grip tape and rotating at a rate of five rotations per minute was used to assess gross locomotor proficiency. The rod was horizontal to the plane of the floor, and the area below the rod was covered with foam strips to cushion falls. Gross kinetic functions were assessed for 5 consecutive days. Each animal was rated for a 1 -min period (excluding the seconds between a completed fall and the replacing of the animal onto the rod). The number of falls observed during the 1-min contact with the rod constituted the gross measure of kinesis.

\section{Visual Learning and Performance}

Discrimination training. Following a 13-day postoperative recovery interval, training began on the 3 -cm-diameter circle $(\mathrm{S}-$ ) versus the $17-\mathrm{cm}$-diameter circle $(\mathrm{S}+)$ discrimination task. Each trial began by placing the rat in the start location with its head facing the front wall of the apparatus. The left-right alley positions of the $\mathrm{S}-$ and $\mathrm{S}+$ stimuli and the escape platform were randomized using a Fellows (1967) series. Correct responses were defined as entry into the arm of the $\mathrm{Y}$ containing the larger stimulus and subsequent escape onto the submerged platform. Errors were defined as entry into the alley containing the smaller diameter circle to the point that the root of the tail crossed the entrance to the arm. Animals were permitted to self-correct incorrect choices, and they were allowed to remain on the platform for approximately $10 \mathrm{sec}$. During each intertrial interval (ITI) of approximately $20 \mathrm{sec}$, the animal was placed in the home cage while the experimenter arranged the stimuli and positioned the escape platform for the next trial. The animals were trained on 10 trials per day, and the criterion to demonstrate acquisition was defined as one session of nine correct trial choices within a sequence of 10 trials. Upon attaining criterion, each animal proceeded with the procedural phases designed to examine relational perception.

Relational discrimination. The probes of relational perception consisted of four phases. As was the case in discrimination training, the escape platform was located beneath the larger of two stimuli in each trial of each phase. In Phase 1, the animals were required to discriminate between stimuli from different categories, wherein the diameters of the circles were reduced from $3 \mathrm{~cm}$ and $17 \mathrm{~cm}$ to $5 \mathrm{~cm}$ and $15 \mathrm{~cm}$, respectively. In the second phase, the stimuli used in training (i.e., the original discrimination) and in Phase 1 were used. Each session required the rat to discriminate between a $3-\mathrm{cm}$ circle $(\mathrm{S}-)$ and a $17-\mathrm{cm}$ circle $(\mathrm{S}+)$, or between a $5-\mathrm{cm}$ circle $(\mathrm{S}-)$ and a 15 -cm circle $(S+)$ for an equal number of trials within each session.
Again, the criterion to demonstrate acquisition was defined as one session of nine correct choices within a session of 10 trials. Phase 3 was composed of two subphases. First, across five sessions composed of 50 trials total, animals were required to discriminate between two stimuli in which the diameters of the circles were reduced to $7 \mathrm{~cm}(\mathrm{~S}-)$ and $13 \mathrm{~cm}(\mathrm{~S}+)$. Next, across another five sessions composed of 50 total trials, animals were required to discriminate between two stimuli in which the diameters of the circles were reduced further to $9 \mathrm{~cm}(\mathrm{~S}-)$ and $11 \mathrm{~cm}(\mathrm{~S}+)$. After testing occurred for 10 sessions, animals completed Phase 3 and then entered Phase 4 , the final relational discrimination phase.

In Phase 4, across seven 18-trial sessions (126 total trials), animals were presented with assorted-paired comparisons, in which both cues originated from within the same category. Hence, each pair contained either only previously negative $(S-)$ circles $(3,5,7$, or $9 \mathrm{~cm})$, or only previously positive $(\mathrm{S}+)$ circles $(11,13,15$, or $17 \mathrm{~cm})$.

Specifically, the animals were required to discriminate between two previously negative circles wherein the larger stimulus was 2,4 , or $6 \mathrm{~cm}$ larger in diameter than the smaller stimulus (e.g., $3 \mathrm{vs} .5 \mathrm{~cm}$, 3 vs. $7 \mathrm{~cm}$, or 3 vs. $9 \mathrm{~cm}$ ). The correct choice was the larger of the two previously negative circles. Similarly, animals were required to discriminate between two previously positive circles wherein the larger stimulus was 2,4 , or $6 \mathrm{~cm}$ larger in diameter than the smaller stimulus (e.g., 15 vs. $17 \mathrm{~cm}, 13$ vs. $17 \mathrm{~cm}$, or $11 \mathrm{vs.} 17 \mathrm{~cm}$ ). Thus in this phase, all trials involved the execution of within-category discriminations. The correct choice was the larger of the two previously positive circles. Within each session of 18 trials, each set of 6 trials was composed of paired comparisons with differences of 2 , 4 , or $6 \mathrm{~cm}$. The order of the assorted comparisons was presented in a quasirandom manner (i.e., 17 vs. 11,9 vs. 3,17 vs. 15,15 vs. 11 , 7 vs. 5,17 vs. 13,9 vs. 7,9 vs. 3,17 vs. 11,9 vs. 5,5 vs. 3,13 vs. 11,7 vs. 3,15 vs. 13,15 vs. 11,17 vs. 11,9 vs. 5 , and 9 vs. 3 ). Following the completion of seven sessions in Phase 4, procedures were terminated temporally for all animals.

Retention. Following a 3-month hiatus, long-term retention was explored by retraining the animal through criterion on the original discrimination.

\section{RESULTS}

\section{Lesion Analysis}

Representative photographs of PS lesions (observed from the dorsal perspective) and TE2 lesions (from the lateral perspective) are displayed in Figure 1. The location of the lesions appeared to be consistent with those depicted in previous investigations (Davis \& McDaniel, 1993; Kolb et al., 1994; McDaniel \& Skeel, 1993; Meyer, Meyer, \& Cloud, 1986).

Microscopic examination of the sectioned brains revealed that PS lesions removed variable amounts of Zilles's (1985) Oc1M and Oc2L, and large portions of Oc2MM and Oc2ML. The anatomical alterations induced by PS lesions are displayed in reconstructions of coronal sections of typical PS lesions in Figure 2. All PS lesions invaded portions of retrosplenial agranular cortex, and 1 PS animal had slight damage to the corpus callosum. Patchy gliosis, cell loss, and cell shrinkage in the lateral geniculate nucleus accompanied PS lesions.

The topography and anatomical alterations induced by TE2 lesions appeared to be similar to those reported in several previous studies (Davis \& McDaniel, 1993; Kolb et al., 1994; McDaniel \& Skeel, 1993). Reconstructions of coronal sections of typical TE2 lesions are shown in 

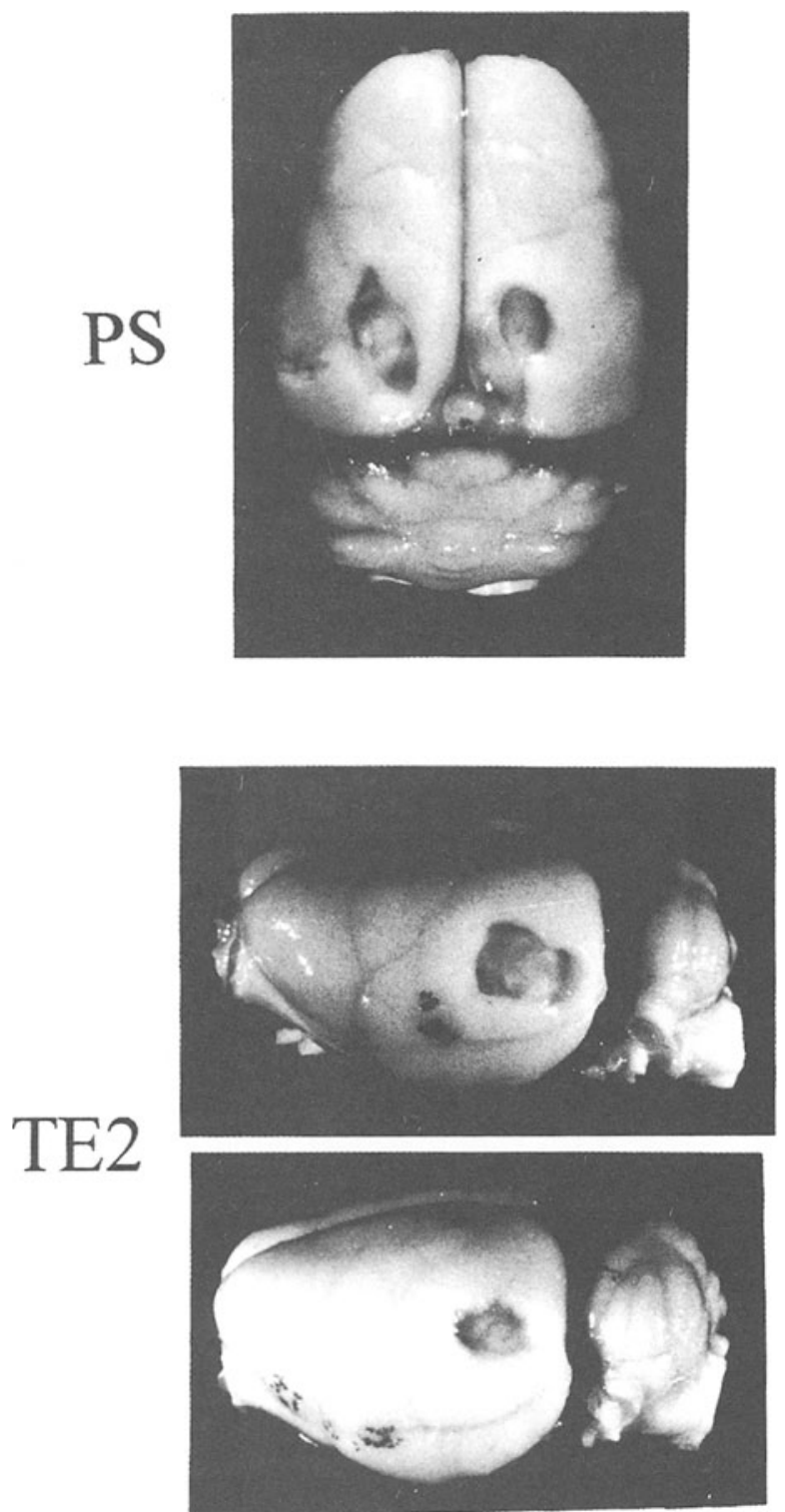

Figure 1. Photographs depicting representative PS and TE2 lesions as viewed from the dorsal and lateral perspectives, respectively.

Figure 3. In most cases lesions invaded large portions of Zilles's (1985) Te2, variable amounts of Te3, the posterior region of Te1, and a modest portion of the lateral border of lateral peristriate cortex (Oc2L). Additionally, lesions in 2 TE2-damaged animals included minor invasion of the perirhinal cortex, a finding also reported by Kolb et al. (1994). In cases of TE2 lesions, as has been described in other studies (Davis \& McDaniel, 1993; Meyer et al., 1986), there was no obvious evidence of necrosis or gliosis within the thalamus. Although there appeared to be no significant subcortical or callosal damage, $1 \mathrm{TE}$ animal did sustain superficial unilateral damage to the ventrolateral portion of the hippocampus.

\section{Rotating Rod Test}

An analysis of variance (ANOVA) for a $3 \times 5$ splitplot factorial (SPANOVA) confirmed that there were no overall differences among the three lesion groups across assessments in terms of number of falls $[F(2,12)=0.20$, $p>.05]$. The analysis also revealed that performances of all groups improved significantly over the course of the five assessments $[F(4,48)=27.94, p<.0001]$. The latter result was due to parallel improvement in locomotion
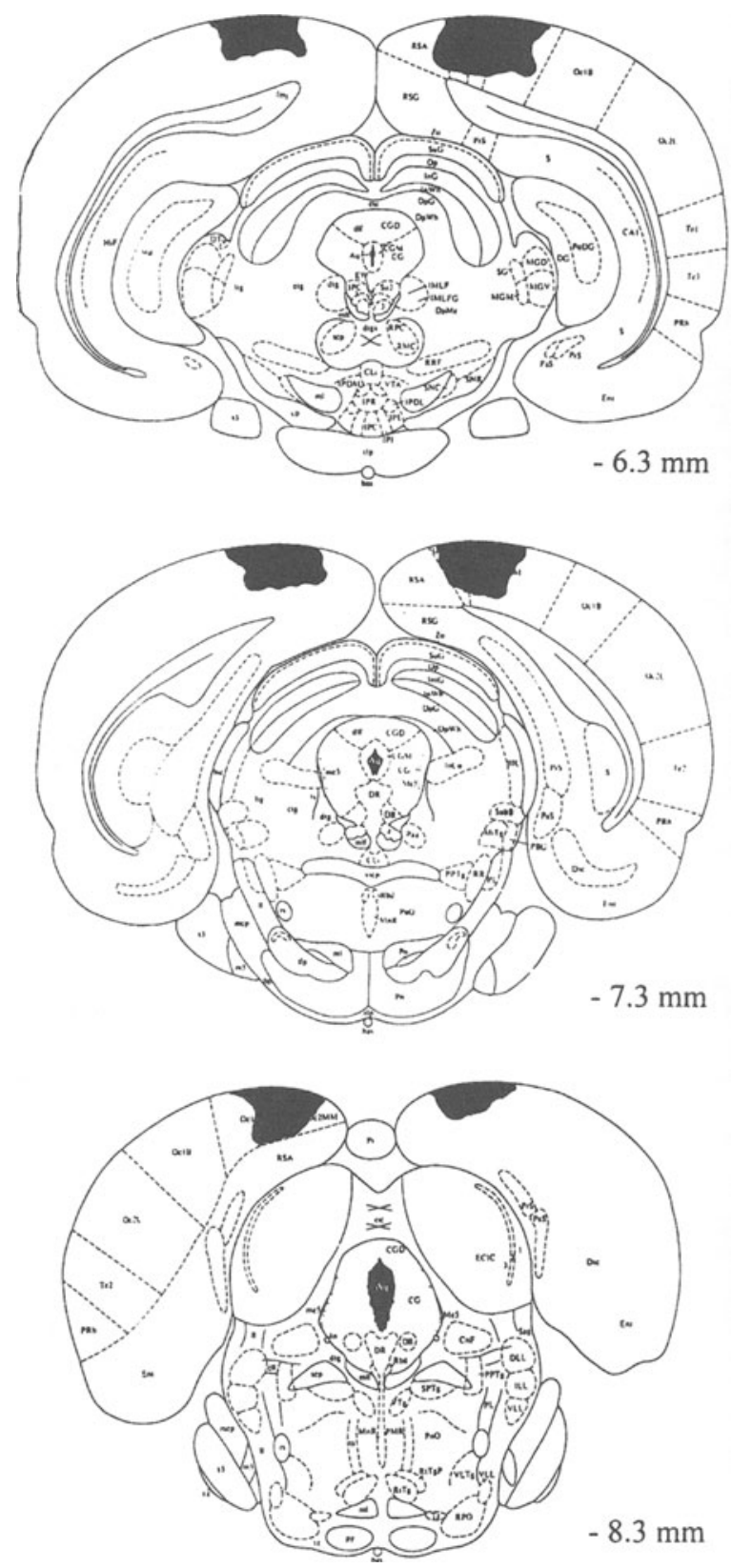

Figure 2. Reconstructions of representative coronal sections depicting anatomical alterations induced by PS lesions. Numerals concern the approximate posterior location with respect to bregma. 

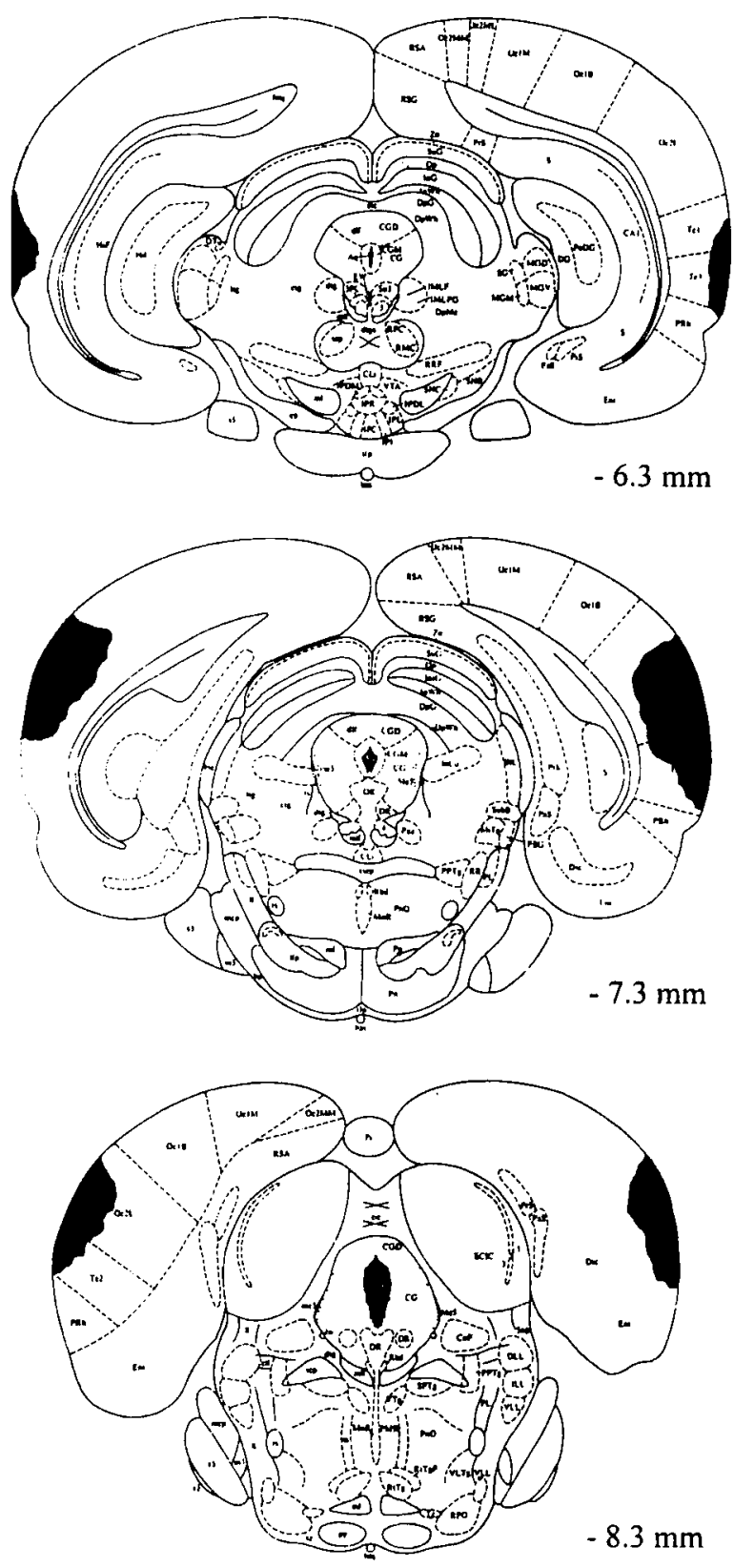

Figure 3. Reconstructions of representative coronal sections depicting anatomical alterations induced by TE2 lesions. Numerals concern the approximate posterior location with respect to bregma.

and balance as a function of exposure to the task and as a function of recovery time for all groups.

\section{Discrimination Training}

A one-way ANOVA was used to evaluate the number of errors through criterion committed by the animals with different lesions while learning the original discrimination (i.e., 3 vs. $17 \mathrm{~cm}$ ). As is apparent in Figure 4, the analysis revealed that all groups learned the initial discrimination task with nearly equivalent proficiency $[F(2,12)=0.40, p>.05]$.

Relational discrimination. A two-way ANOVA was used to assess the number of errors committed through criterion on the original discrimination relative to the number of errors on the discrimination used in Phase 1 of relational performance testing. The analysis revealed that the number of errors committed on the second discrimination was significantly lower than that observed for the original $\operatorname{task}[F(1,12)=101.29, p<.0001]$. This result, displayed in Figure 4, shows that the animals transferred from the first pair of stimuli to the second pair of stimuli with relative ease. The interaction between lesion and discrimination problems was nonsignificant $[F(2,12)=$ $0.33, p>.05]$. This finding reveals that the transfer of training was equivalent for the three lesion groups.

A one-way ANOVA was used to examine errors through criterion across groups when paired stimuli, differing by $14 \mathrm{~cm}$, were interspersed with an equal number of trials of paired stimuli differing by $10 \mathrm{~cm}$ (e.g., Phase 2). Shown graphically in Figure 4, satisfactory performance by all animals was found on this problem. No differences among the lesion groups were observed $[F(2,12)=0.66$, $p>.05]$.

Next, a $3 \times 2$ SPANOVA was used to analyze the proportion correct responses for when the difference between paired stimuli was reduced by two more steps. That is, 50 trials were administered in which stimuli differed by $6 \mathrm{~cm}$ (i.e., $7 \mathrm{vs} .13 \mathrm{~cm}$ ); then, 50 trials were administered in which stimuli differed by $2 \mathrm{~cm}$ (i.e., $9 \mathrm{vs} .11 \mathrm{~cm}$ ). As before, no differences among the lesion groups emerged on these discriminations $[F(2,12)=1.01, p>.05]$. However, greater choice accuracy was observed on the 7-vs. 13-cm discrimination (6-cm difference between stimuli; $80 \%$ choice accuracy for all groups) than on the 9- vs. $11-\mathrm{cm}$ discrimination (2-cm difference between stimuli; range of $55 \%$ to $60 \%$ correct for all groups) $[F(1,12)=$ $45.84, p<.0001]$. The interaction between lesion and discrimination problems was nonsignificant $[F(2,12)=$ $0.67, p>.05]$.

Finally, a two-way SPANOVA ( 3 lesion groups $\times 3$ steps of difference between paired stimuli) was used to assess the proportion correct responses among all lesion groups across 126 trials defined earlier as Phase 4, wherein stimuli were randomly presented from within one category or the other. Specifically, animals were given an equal number of trials in which stimuli paired from within the same category differed by $6 \mathrm{~cm}$ (e.g., $3 \mathrm{vs.} 9 \mathrm{~cm}$ and 11 vs. $17 \mathrm{~cm}$ ), $4 \mathrm{~cm}$ (e.g., 13 vs. $17 \mathrm{~cm}, 11$ vs. $15 \mathrm{~cm}, 5$ vs. $9 \mathrm{~cm}$, and 3 vs. $7 \mathrm{~cm}$ ), and $2 \mathrm{~cm}($ e.g., 15 vs. $17 \mathrm{~cm}, 13$ vs. $15 \mathrm{~cm}, 11$ vs. $13 \mathrm{~cm}, 7$ vs. $9 \mathrm{~cm}, 5$ vs. $7 \mathrm{~cm}$, and 3 vs. $5 \mathrm{~cm}$ ). This analysis failed to reveal a significant main effect of lesion $[F(2,12)=1.42, p>.05]$ or the interaction between lesion and step difference $[F(4,24)=0.44, p>$ $.05]$. However, an overall significant difference was found among problems $[F(2,24)=9.86, p<.001]$. This result, shown in Figure 5, reflects the overall superior performance of all groups on discriminations with pairs 


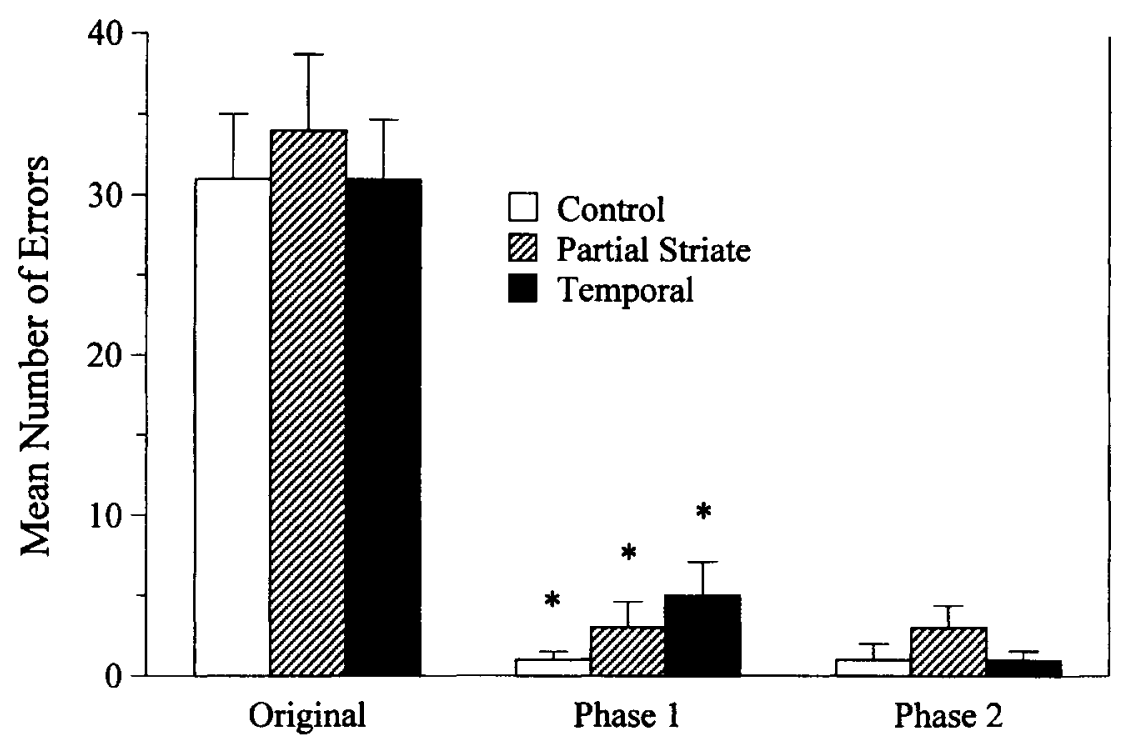

\section{Discrimination Condition}

Figure 4. Mean and standard error number of errors committed through criterion across groups for the original discrimination and for subsequent conditions testing relational judgments. Asterisks indicate significant performance differences between the first and second tasks for all groups.

in which stimuli differed by $6 \mathrm{~cm}(M=.68, S D=0.10)$ relative to pairs in which stimuli differed by $4 \mathrm{~cm}(M=.58$, $S D=0.09)$ or by $2 \mathrm{~cm}(M=.52, S D=0.10)$.

\section{Microanalysis of Relational Discriminations}

Wilson and DeBauche (1981) reported that monkeys with bilateral lesions of the IT cortex, when presented with between-category discriminations, exhibited greater performance deficits than did normal monkeys or monkeys with PS lesions. As was the case in the Wilson and DeBauche investigation, the original discrimination of the present experiment involved comparing the extreme values from a given continuum of stimuli. Circles ranged from 3 to $17 \mathrm{~cm}$ in diameter by increments of $2 \mathrm{~cm}$. The continuum was, in essence, divided into two categories wherein stimuli with diameters greater than $10 \mathrm{~cm}$ were considered to be similar to the original positive stimulus. Likewise, stimuli with diameters smaller than $10 \mathrm{~cm}$ were considered to be more closely related to the original negative stimulus. Thus, between-category discriminations involved 7-versus $13-\mathrm{cm}$ - and 9-versus $11-\mathrm{cm}$-diameter circles. In contrast, within-category judgments utilized stimuli such as 11 versus $17 \mathrm{~cm}$ or 3 versus $5 \mathrm{~cm}$, where both members of the paired comparison originated from the same side of the continuum. Although the same physical step differences between compared stimuli $(2-\mathrm{cm}$ step difference and 6-cm step difference) were represented by both judgment types, the distinction between the judgment types was that the between-category judgment compared a stimulus that approximated or was equivalent to the original positive cue, with another stimulus that was similar to the previously experienced negative stimuli. On the other hand, the within-category judgment involved the comparison of two stimuli, both of which were near or equivalent to either the original positive cue or the original negative cue.

In order to compare discrimination performances among groups on between-category stimuli with discrimination performances among groups on within-category stimuli, data from training and performance testing were combined to form between-category and within-category problems. Further, in both considerations, the diameters of the stimuli differed by either 6 or $2 \mathrm{~cm}$. In order to evaluate relational learning, the pairs of stimuli included in the statistical analysis were those in which performance on a given physical step difference could be assessed for both types of judgment for all animals. (In other words, 4-cm differences were not examined in the phases of training and testing that involved between-category judgments.) A comparison on within- versus between-category performance was central to the analyses conducted by Wilson and DeBauche (1981), and it was on the betweencategory discriminations where significant deficits were observed in the IT-lesioned monkeys. Importantly, all animals performed poorly on within-category discriminations, as indicated by the quote: "Finally, it should be noted that the animals with IT lesions performed as well (or as poorly) on within-category discriminations as animals in the other groups" (p. 35).

A three-way SPANOVA with two repeated measures was used to examine the proportion of correct responses of animals as a function of lesion condition (i.e., 3 lesion 


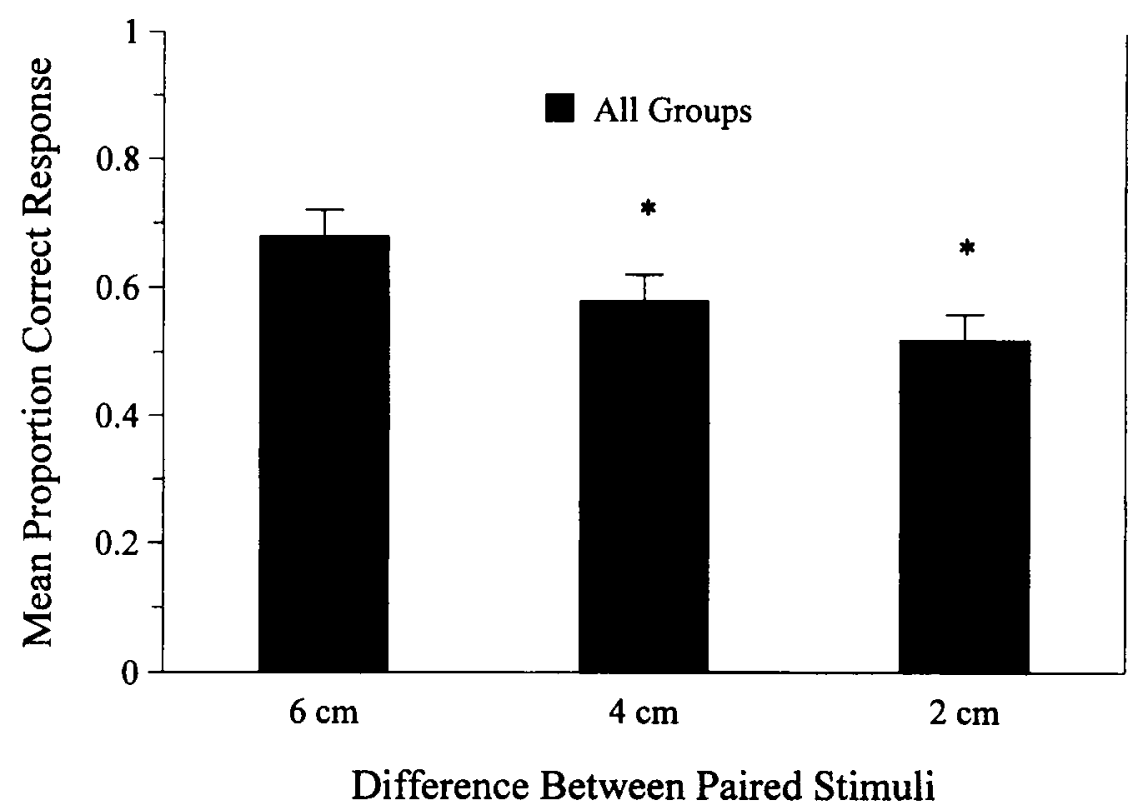

Figure 5. Mean and standard error proportion of correct responses across lesion groups for within-category stimuli differing by 6,4 , or $2 \mathrm{~cm}$. Asterisks indicate significant decrease in accuracy for all lesion groups with stimulus pairs differing by 4 and $2 \mathrm{~cm}$.

groups $\times 2$-vs. 6-cm physical step differences $\times$ betweenvs. within-category problems). This analysis failed to reveal influences that could be ascribed to the lesions (i.e., for the main effects and all interactions involving the lesion condition, $p \mathrm{~s}>.05$ ). However, as expected, a significant main effect was found for the physical step difference between stimuli $[F(1,12)=92.38, p<.0001]$. That is, all groups performed much more accurately on trials in which stimuli differed by $6 \mathrm{~cm}(M=.74, S D=.02)$ than on trials in which stimuli differed by $2 \mathrm{~cm}(M=.52, S D=$ .02 ). The results are illustrated in Figure 6. It is apparent that there is a floor effect wherein the performance of all groups plummeted to chance when animals were required to discriminate stimuli differing by a $2-\mathrm{cm}$ diameter. This is probably due to the rat's limited visual acuity relative to some animals (Dean, 1990). In addition, a significant main effect was observed for between-category versus within-category judgments $[F(1,12)=9.00, p=$ $.0111]$. This result showed that, regardless of lesion group, overall the animals performed more accurately when discriminating between pairs involving stimuli from between categories $(M=.66, S D=.01)$ than when discriminating between pairs involving cues from within the same category $(M=.61, S D=.02)$. The category problem $X$ step difference interaction was not significant.

\section{Retention}

A one-way ANOVA was used to analyze the savings scores across lesion groups when the animals were retrained on the original visual discrimination (i.e., 3 vs. $17 \mathrm{~cm}$ ) after a 3-month hiatus from training. No differ- ences emerged among lesion groups on this discrimination $[F(2,12)=0.5625, p>.05]$. In fact, considerable savings were observed for animals in all three groups $(M=$ $.81, S D=.24)$.

\section{DISCUSSION}

The influences of lesions within the visual region of the rat's temporal cortex (TE2) or striate cortex on visual relational perception were investigated. Initially rats were trained to discriminate between a $17-\mathrm{cm}$-diameter circle and a 3-cm-diameter circle. Consistent with previous results (Davis \& McDaniel, 1993; Meyer et al., 1986), rats with TE2 lesions learned this simple visual discrimination as rapidly as normal controls. A similar result has been observed in studies with IT-injured monkeys. That is, although monkeys with IT lesions must relearn visual discriminations acquired prior to brain damage (Dean \& Cowey, 1977; Dean \& Weiskrantz, 1974), they learn and perform simple visual discriminations learned postoperatively as well as normal control animals (e.g., Butter \& Hirtzel, 1970; Ungerleider \& Pribram, 1977). With respect to the present study, once the initial discrimination was leàrned, the difference in diameter between the two cues was reduced in a series of steps. Thereafter, the animals were tested for their abilities to discriminate other pairings of stimuli in accordance with a relational rule (i.e., navigating toward a larger circle led to escape, whereas navigating toward a smaller circle did not). Contrary to monkeys with IT lesions (Wilson \& DeBauche, 1981), in the present study rats with TE2 lesions trans- 


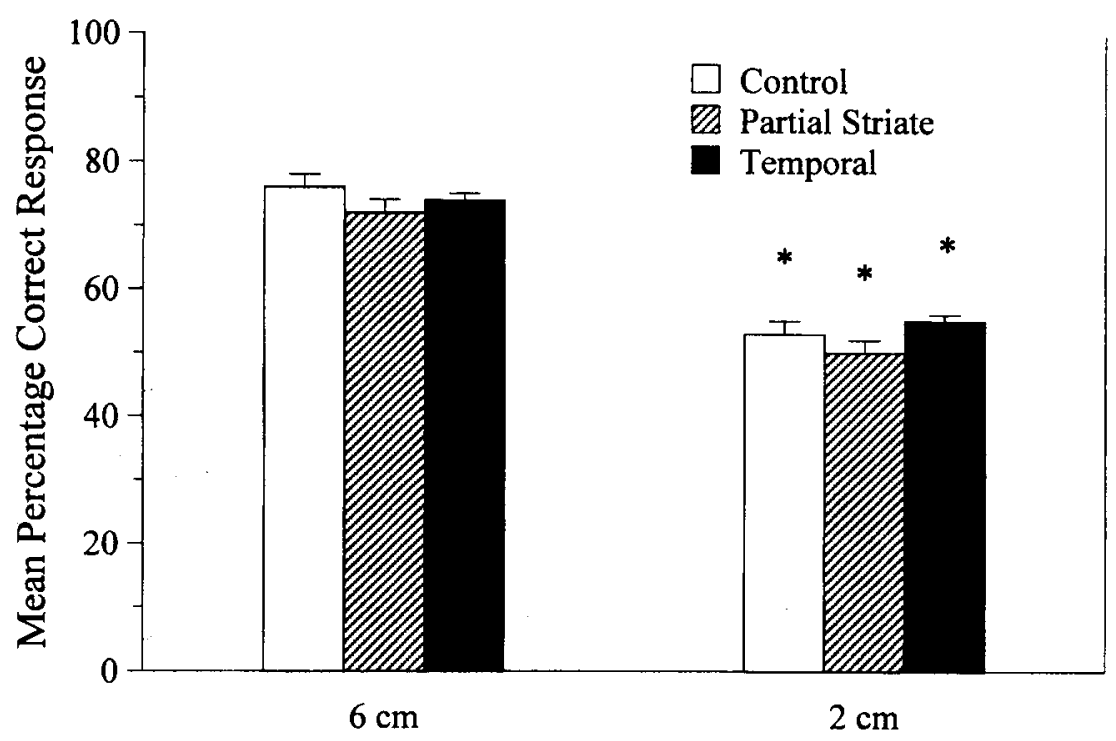

\section{Difference Between Paired Stimuli}

Figure 6. Mean and standard error percentage correct responses, pooled across between-category and within-category judgments for stimuli differing by 6 and $2 \mathrm{~cm}$. Asterisks indicate significantly better performance on trials with stimuli differing by $6 \mathrm{~cm}$.

ferred relatively well across all between-category problems demanding relational perception. Consistent with the Wilson and DeBauche study with monkeys, we found that performance was significantly better for all groups when challenged with between-category discriminations than with within-category discriminations. It was on between-category judgments that monkeys with IT lesions performed poorly relative to controls and, as indicated earlier, both brain-injured and normal monkeys performed poorly on the within-category discriminations. Finally, following a 3-month interval, the rats were retrained on the original discrimination to examine retention. Both the normal controls and the brain-injured animals showed good savings. This result is similar to that of Myhrer (1992), who found, as has been demonstrated with IT-injured primates, that rats with temporal cortex lesions have retrograde rather than anterograde retention impairments. As expected, rats with PS lesions performed well on all problems. Similar results were reported for monkeys with partial PS lesions (Wilson \& DeBauche, 1981).

There are several possible reasons why the rats with TE2 lesions performed well in the present study, but monkeys with IT lesions in the Wilson and DeBauche (1981) study performed poorly with the relational or categorical aspects of testing. First, Wilson and DeBauche trained their animals on the discrimination prior to surgery and then retrained them on the same discrimination postsurgically prior to testing them with combinations of intermediate stimuli. All training in the present study was conducted postsurgically. Second, and perhaps more importantly, after learning the original discrimination, our animals were presented with a series of transfer trials with stimuli differing from the original stimuli by a small amount. Specifically, the 17 - versus $3-\mathrm{cm}$ discrimination was shifted to a 15 - versus $5-\mathrm{cm}$ discrimination, and after attaining criterion with these stimuli, the animals experienced trials involving both pairs of stimuli. This experience may have facilitated the use of a relational rule by the rats in the present study. In contrast, Wilson and DeBauche presented the trials with numerous intermediate stimuli immediately after retraining the animals on the original discrimination postsurgically. Results more consistent with those observed by Wilson and DeBauche may have been observed if we had used an identical training protocol. We believed that the result observed in monkeys was robust. Thus, we expected that similar results would be observed in rats prepared with TE2 lesions despite the use of behavioral procedures that had been developed to optimize transfer of training.

Another plausible explanation can be found in a recent review of the literature concerning the dual cortical visual system hypothesis from a comparative neurological perspective (Ellard, 1998; see especially p. 179 for the quotations below). Ellard reported that in primates some regions of the ventral "stream" (i.e., posterior temporal cortex) are specialized for "movement-based imagery." That is, some temporal regions play a prominent role in "viewpoint-invariant representation of threedimensional objects." Given the results in our lab (Davis \& McDaniel, 1993) and Kolb's lab (Kolb et al., 1994) showing that deficits in visual memory accompany TE2 lesions in rats, we suspect that this may apply to two- 
dimensional forms as well. Ellard also postulated that other temporal association areas "are perhaps more concerned with more abstract, cognitive representations of objects." Ellard speculated that the ventral stream identified in nonprimate species subserves only the former function, and that it is only in primates that the latter functions have been subsumed by the temporal cortex. The data from the present study are consistent with Ellard's hypothesis.

Obviously more research is needed to resolve this topic, but it is clear that in the present study rats with TE2 lesions generalized a relational perceptual rule relatively well across the various pairs of circular stimuli with intermediate diameters. It may be the case that perceptual categorization of the type studied here is subserved by other visual cortical territories in animals with less complexly organized temporal lobes. A likely candidate region mediating such basic functions in nonprimate mammals would be the one of the many retinotopically organized regions adjacent to TE2, namely the lateral peristriate cortex (see, e.g., Montero, 1993). Although the influences of total lateral peristriate cortex lesions on visual discrimination have been studied (Gallardo et al., 1979; McDaniel, Coleman, \& Lindsay, 1982; McDaniel \& Wall, 1988), to our knowledge, neurobehavioral studies of distinct subterritories within the lateral peristriate cortex have not yet been conducted.

It is hoped that the results of this study will stimulate other investigations of the roles played by rodent TE2 in higher perceptual functions. We plan to replicate this study using stimuli that control luminous flux as potential discriminanda and behavioral protocols parallel to the relational perception testing methods used by Wilson and DeBauche (1981).

\section{REFERENCES}

BUtTER, C. R., \& HIRTZEL, M. (1970). Impairment in sampling visual stimuli in monkeys with inferotemporal lesions. Physiology \& Behavior, 5, 369-370.

Davis, B. K.. \& MCDANiEL, W. F. (1993). Visual memory and visual spatial functions in the rat following parietal and temporal cortex injuries. Physiology \& Behavior, 53, 145-151.

DEAN, P. (1990). Sensory cortex: Visual perceptual functions. In B. Kolb \& R. C. Tees (Eds.), The cerebral cortex of the rat (pp. 275. 307). Cambridge, MA: MIT Press.

DEAN, P., \& COWEY, A. (1977). Inferotemporal lesions and memory for pattern discriminations after visual interference. Neuropsychologia, 15, 93-98.

Dean, P. \& Weiskrantz, L. (1974). Loss of preoperative habits in thesus monkeys with inferotemporal lesions: Recognition failure or relearning deficit? Neuropsychologia, 12, 299-311.

EllaRD, C. G. (1998). Comparative perspectives on multiple cortical visual systems. Neuroscience \& Biobehavioral Reviews, 22, 173-180.

Fellows, B. J. (1967). Chance stimulus sequences for discrimination tasks. Psychological Bulletin, 67, 87-92.

Gallardo, L., Mottles, M., Vera, L., Carrasco, M. A., Torrealba, F., Montero, V. M., \& Pinto-HAMUY, T. (1979). Failure by rats to learn a visual conditional discrimination after lateral peristriate cortical lesions. Physiological Psychology, 7, 173-177.

Haxby, J. V., Grady, C. L., Horwitz, B., Ungerleider, L. G.,
Mishioin, M., Carson, R. E., Herscovitch, P., Schapiro, M. B., \& RAPOPORT, S. I. (1991). Dissociation of object and spatial visual processing pathways in human extrastriate cortex. Proceedings of the National Academy of Science, 88, 1621-1625.

Horwitz, B., Grady, C. L., Haxby, J. V., Schapiro, M. B., Rapoport, S. I., UNGerleider, L. G., \& MishKIN, M. (1992). Functional associations among human posterior extrastriate brain regions during object and spatial vision. Journal of Cognitive Neuroscience, 4, 311 322.

Hughes, H. C. (1977). Anatomical and neurobehavioral investigations concerning the thalamocortical organization of the rat's visual system. Journal of Comparative Neurology, 175, 311-335.

KolB, B. (1990). Posterior parietal and temporal association cortex. In B. Kolb \& R. C. Tees (Eds.), The cerebral cortex of the rat (pp. 459. 471). Cambridge, MA: MIT Press.

Kolb, B., Buhrmann, K., McDonald, R., \& Sutherland, R. J. (1994). Dissociation of the medial prefrontal, posterior parietal, and posterior temporal cortex for spatial navigation and recognition memory in the rat. Cerebral Cortex, 6, 664-680.

KOLB, B., \& WALKEY, J. (1987). Behavioural and anatomical studies of the posterior parietal cortex in the rat. Behavioural Brain Research, 23, 127-145.

KolB, B., \& WhiSHAW, I. Q. (1990). Fundamentals of neuropsychology (3rd ed.). New York: W. H. Freeman.

Macko, K. A., Jarvis, C. D., Kennedy, C., Miyaoka, M., Shinohara, M., Sokoloff, L., \& Mishkin, M. (1982). Mapping the primate visual system with [2-14C]deoxyglucose. Science, 218, 394397.

MCDaniel, W. F., Coleman, J., \& Lindsay, J. F., JR. (1982). A comparison of lateral peristriate and striate neocortical ablations in the rat. Behavioural Brain Research, 6, 249-272.

MCDANIEL, W. F., \& NoBle, L. M. (1984). Visual pattern discrimination following bilateral striate or lateral peristriate neocortical injuries. IRCS Medical Science Research, 12, 1084-1085.

MCDANIEL, W. F., \& SKeEL, R. L. (1993). Visual-spatial functions persist following temporal and posterior parietal cortex lesions in rat. Physiology \& Behavior, 53, 199-203.

MCDANIEl, W. F., \& Thomas, R. K. (1978). Temporal and parietal association cortex lesions and spatial and black-white reversal learning in the rat. Physiological Psychology, 6, 300-305.

McDaniel, W. F., Via, J. D., Smith, J. S., Wells, D. L., Fu, J. J. BisHOP, J. F., BoYd, P. A., \& LedesMA, H. M. (1995). Unilateral injury of posterior parietal cortex and spatial learning in hooded rats. $B e$ havioural Brain Research, 70, 165-179.

MCDANIEL, W. F., \& WALL, T. T. (1988). Visuospatial functions in the rat following injuries to striate, peristriate, and parietal neocortical sites. Psychobiology, 16, 251-260.

McDaniel, W. F., Williams, L. B., Attaway, C. M., \& Compton, D. M. (1998). Turn-signal utilization by rats with either unilateral or bilateral posterior parietal cortex injuries. Psychobiology, 26, 143152

Meyer, P. M., Meyer, D. R., \& Cloud, M. D. (1986). Temporal neocortical injuries in rats impair attending but not complex visual processing. Behavioral Neuroscience, 100, 845-851.

MyHRER, T. (1992). Selective lesions in the temporal-hippocampal region of the rat: Effects on acquisition and retention of a visual discrimination task. Behavioral \& Neural Biology, 58, 8-15.

Mishkin, M., Ungerleider, L. G., \& Macko, K. A. (1983). Object vision and spatial vision: Two cortical pathways. Trends in Neurosciences, 6, 414-417.

MonTero, V. (1993). Retinotopy of cortical connections between the striate cortex and extrastriate visual areas in the rat. Experimental Brain Research, 94, 1-15.

ShI, C.-J., \& CASSELL, M. D. (1997). Cortical, thalamic, and amygdaloid projections of rat temporal cortex. Journal of Comparative Neumlogy, 382, 153-175.

SoвotKA, S., \& RiNGo, J. L. (1993). Investigations of long-term recognition and association memory in unit responses from inferotemporal cortex. Experimental Brain Research, 96, 28-38. 
Ungerleider, L. G., \& MishKin, M. (1982). Two cortical visual systems. In D. J. Ingle, M. A. Goodale, \& R. J. W. Mansfield (Eds.), Analysis of visual behavior (pp. 549-586). Cambridge, MA: MIT Press.

Ungerleider, L. G., \& Pribram, K. L. (1977). Inferotemporal versus combined pulvinar-prestriate lesions in the rhesus monkey: Effects on color, object, and pattern discriminations. Neuropsychologia, 15, $481-498$.

Wilson, M., \& DeBauche, B. A. (1981). Inferotemporal cortex and categorical perception of visual stimuli by monkeys. Neuropsychologia, 19, 29-41.

Zhu, X. O., Brown, M. W., \& Aggleton, J. P. (1995). Neuronal sig- nalling of information important to visual recognition memory in rat rhinal and neighbouring cortices. European Journal of Neuroscience, 7, 753-765.

Zhu, X. O., McCabe, B. J., Aggleton, J. P., \& Brown, M. W. (1996). Mapping visual recognition memory through expression of the immediate early gene c-fos. NeuroReport, 7, 1871-1875.

ZiLLes, K. (1985). The cortex of the rat: A stereotaxic atlas. Berlin: Springer-Verlag.

(Manuscript received September 23, 1998; revision accepted for publication December 2,1998.) 